\title{
REVIEW
}

For reprint orders, please contact: reprints@futuremedicine.com

\section{Is carbonic anhydrase IX a validated target for molecular imaging of cancer and hypoxia?}

\author{
Jianbo Li ${ }^{\ddagger 1,2}$, Guojian Zhang ${ }^{\ddagger 1,2}$, Xuemei Wang ${ }^{*, 1}$ \& Xiao-Feng Li ${ }^{*, 2}$
}

\begin{abstract}
The presence of hypoxia is a general feature of most solid malignancies, and hypoxia is considered as one of major factors for anticancer therapy failure. Carbonic anhydrase IX (CAIX) has been reported to be an endogenous hypoxia marker, CAIX monoclonal antibodies, their segments and inhibitors are developed for CAIX imaging. However, growing evidence indicates that CAIX expression under hypoxia condition may be cancer cell lines or cancer-type dependent. Here we review the current literature on CAIX and discuss the advantage and limitation of CAIX as a target for tumor hypoxia imaging. Accordingly, CAIX would be unreliable as a universal target for cancer and tumor hypoxia visualization.
\end{abstract}

In 2013, approximately 1,660,290 new cancer cases are diagnosed and 580,350 patients are died of cancer in the USA [1]. Hypoxia is a common feature of large primary solid malignancies and is recognized as a negative determinant of clinical outcome [2-9]. It is now established that tumor hypoxia is an important determinant of relapse-free survival and overall clinical outcome [4,10-11]. This appears to be largely independent of the treatment modality used and implies that hypoxia is associated with a more aggressive tumor phenotype. There seems to be at least three distinct manifestations of the hypoxic phenotype: Hypoxic cells are more resistant to ionizing radiation and chemotherapy than aerobic cells; The hypoxic environment is selective for genetically unstable tumor cells, and hypoxia is associated with tumors that are more likely to metastasize [10,12-13]; and hypoxia regulates the level of HIF- $1 \alpha$, which controls the expression of genes that are involved in oxygen and glucose supply and utilization [14]. Therefore, it is important to detect the hypoxic status for solid cancers.

Recently multiple biomarkers related to tumor hypoxic microenvironment have been discovered and offered as targets for cancer detection, treatment and monitoring, such as HIF-1 [15-17], VEGF and VEGF receptors [18-20], glucose transporter 1 [21-23] and carbonic anhydrase IX (CAIX) [24-29]. CAIX is strongly induced by hypoxia through the HIF-1 transcription factor, and has reported to overexpress in some types of cancer [30,31]. Promising results for using anti-CAIX antibodies and CAIX inhibitors for tumor hypoxia imaging have been reported [32-34].

However, growing evidence indicates that the CAIX expression under hypoxia conditions in cancer cell lines is cancer cell lines or cancer types dependent [30], and some cancer cell lines have undetectable CAIX expression under hypoxia condition [35,36]. In addition, re-oxygenated (previously hypoxic) cancer cells may still show high level of CAIX expression [36]; CAIX has a half-life up to 2-3 days under normoxic condition. In contrast, acute hypoxic cancer cells may not express detectable CAIX.

'Department of Nuclear Medicine, Inner Mongolia Medical University Affiliated Hospital, Hohhot, Inner Mongolia, China 2Department of Diagnostic Radiology, University of Louisville, 530 S Jackson Street, CCB-C07, Louisville, KY 40202, USA

*Authors for correspondence: Tel.: +1 502217 8285; Fax: +1 502852 1754; linucmed@gmail.com; wangxuemei201010@163.com ${ }^{\ddagger}$ Authors contributed equally

\section{KEYWORDS}

- cancer • carbonic anhydrase IX $\bullet$ hypoxia

- molecular imaging 
The open question is whether CAIX is a reliable and universal target for cancer treatment and especially tumor hypoxia visualization. In this review, we focus on the topic of whether CAIX is a validated target for molecular imaging of cancer and hypoxia.

\section{Carbonic anhydrases}

Carbonic anhydrases (CAs) are a large family of zinc metalloenzymes that catalyze the reversible interconversion of carbon dioxide and water to bicarbonate and a proton. There are 16 different alpha-CA isoforms currently known, differing in tissue distribution, subcellular localization and molecular biophysical properties. They are involved in diverse physiological processes, including acid-base regulation, respiration, ion transport and bone resorption [37].

CAIX, a CAs family member, is a transmembrane protein and was first detected in human cervix carcinoma HeLa cells and initially named the MN protein in 1992 by Pastoreková et al. [38] In 1994, Pastorek et al. identified CAIX by sequence analysis of its cDNA isolated using the monoclonal antibody M75 specific for a plasma membrane antigen in HeLa cells [39].

The overexpression of CAIX in normal human tissues is rare [40]: it has limited expression and distribution in a few normal tissues (mainly the GI tract), and this expression is either decreased or lost during carcinogenesis [41]; however, it is ectopically expressed in numerous tumors which are mostly derived from tissues that do not normally express CAIX [25,27-29]. For example, CAIX is overexpressed in clear-cell renal cell carcinoma because of a mutation in Von HippelLindau tumor suppressor, but is expressed at low levels in normal kidney tissue. [42]. This atypical differential expression pattern enables researchers to designate CAIX as a tumor-associated protein and to use it as a biomarker of tumor.

CAIX is a membrane-associated glycoprotein [43], consisting of an extracellular catalytic domain, a proteoglycan-like region (PG domain), a transmembrane anchor and a short C-terminal cytoplasmic tail (Figure 1). The PG domain, which is adjacent to catalytic domain and involves in $\mathrm{CO}_{2}$ hydration at more acidic $\mathrm{pH}$ values, is the distinctive feature of CAIX differing from the other known CAs. Cancer cells maintain a near neutral intracellular $\mathrm{pH}$ by CAIX, thereby favoring cell-survival. The acidic extracellular environment activates metalloproteinases and release of growth factors, thus facilitating tumor invasion and metastases [44]. Pastoreková et al. [45] found CAIX also has a potential to decrease cell-cell adhesion by destabilization of E-cadherin links to cytoskeleton. In hypoxic circumstances, de-adhesion capacity and interaction with $\beta$-catenin may represent one important aspect of CAIX functionality, by which it may contribute to the acquisition of increased tumor aggressiveness.

\section{CAIX \& hypoxia}

Hypoxia is a characteristic feature of many solid tumors [46] and has been described in a variety of human malignancies, including prostate cancer [47], head and neck tumors [48], non-smallcell lung cancer [49], brain cancer [50] and breast cancer [51]. The hypoxic microenvironment is mainly due to an imbalance between oxygen supply and consumption, which is caused by the rapid proliferation of tumor cells resulting in increased diffusion distances between blood microvasculature and the tumor cells [31,52]. The impact of the hypoxic tumor microenvironment is multifaceted [46,53-54], including genetic instability, abnormal angiogenesis, limitless proliferative potential, evasion of apoptosis, invasiveness, survival and metabolism. Hypoxia-inducible factor HIF-1 has been verified as the main transcription factor that regulates the tumor cellular adaptation to hypoxia [55,56].

HIF-1 regulates the expression of many genes under hypoxic conditions through HIF-1 pathway [55-58]. HIF-1 is a heterodimer composed of HIF- $1 \alpha$ and HIF- $1 \beta$ subunit. HIF- $1 \beta$ is constitutively expressed, insensitive to changes in oxygen concentration, while HIF-1 $\alpha$ is very sensitive to oxygen concentration. Under normoxic condition, HIF-1 $\alpha$ undergoes proline hydroxylation and binds to Von Hippel-Lindau protein and is ultimately broken down after ubiquitination. Under hypoxic condition, HIF-1 $\alpha$ is stabilized and translocated to the nucleus and heterodimerizes with constitutively expressed HIF-1 $\beta$. The heterodimer then binds to the hypoxia-response elements sites of target genes, including CAIX, crucial to adapt to the hypoxic environment for the tumor cells. CAIX has thus been proposed as a reliable endogenous hypoxic tumor marker that may be helpful in clinical practice [27].

\section{CAIX-targeted preclinical imaging}

The rare distribution of CAIX in normal tissues makes radionuclide labeled monoclonal 


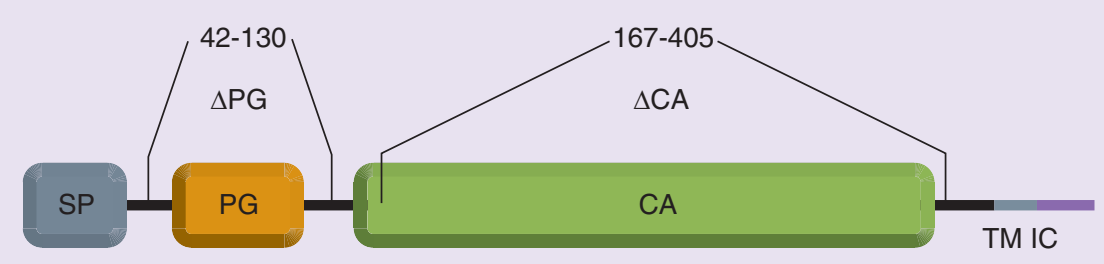

Figure 1. Domain organization of the carbonic anhydrase IX protein.

CA: Catalytic domain; IC: Intracellular cytosolic tail; PG: Proteoglycan-like domain; SP: Signal peptide; TM: Transmembrane segment.

Reproduced with permission from: original published work by De Simone G, Supuran CT [43] ๔ by

Elsevier BV (2010).

antibodies against CAIX or CAIX-specific small molecule inhibitors excellent biomarkers of hypoxia in many solid tumors in preclinical studies $[25,59]$.

Zhang et al. [24] found that ${ }^{125}$ I-labeled monoclonal antibody against CAIX (125I-MAb) could be used to visualize tumor hypoxia. The biodistribution at $24 \mathrm{~h}$ postinjection (p.i.) of ${ }^{125} \mathrm{I}$ $\mathrm{MAb}$ and planar imaging at $48 \mathrm{~h}$ p.i. showed specific tumor uptake in the mouse bearing xenografts of human colorectal cancer HT29 cells. Immunohistochemical staining confirmed prenecrotic overexpression of CAIX within the tumors. Carlin et al. [33] investigated the efficiency of intact and IgG fragments of cG250 against CAIX in mice with HT29 tumor and demonstrated that ${ }^{111}$ In-DO3A-F $\left(\mathrm{ab}^{\prime}\right)_{2}$-cG250, a lower molecular weight antibody fragment, had a faster uptake into areas of high CAIX expression, but had a much lower absolute tumor uptake and reduced tumor to muscle ratios at 24 h p.i. ${ }^{111}$ In-DO3A-Fab-cG250, another fragment, had a similar nature with $\mathrm{F}\left(\mathrm{ab}^{\prime}\right)_{2}$, fragment in vivo. However, highest HT29 tumor uptake of intact antibody ${ }^{111} \mathrm{In}-\mathrm{DO} 3 \mathrm{~A}-\mathrm{cG} 250$ happened 7 days p.i. Accordingly, radionuclide labeled antiCAIX antibodies or IgG fragment could significantly concentrate in the high CAIX expressed HT29 tumors and maybe useful for cancer and hypoxia imaging in this specific cancer line. Of note, hypoxic cancer cells, including HT29 cells, had be observed to have a short lifespan of approximately 2 days in vivo [60] though in vitro cell culture studies demonstrated that hypoxic tumor cells can survive up to approximately 10 days at hypoxic/anoxic atmosphere [61-64]. Therefore, imaging conducted 2-7 days after inoculation of radiolabeled CAIX antibodies or IgG fragments unlikely reflect real time tumor hypoxia.
CAIX inhibitor imaging has been well documented in literature. Akurathi et al. [65] synthesized $\left.{ }^{\left[9{ }^{\mathrm{m}} \mathrm{Tc}\right.}(\mathrm{CO})_{3}(\mathrm{~L})\right](\mathrm{L}=\mathrm{N}$ - (pyridin-2yl-methyl)-N[2-(4-sulfamoylphenyl)-ethyl] ami-

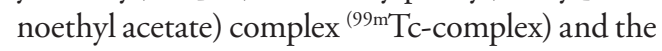
corresponding rhenium congener (Re-complex). In vitro affinity studies revealed that Re-complex had a dissociation constant (Ki) of $58 \mathrm{nM}$ for CAIX. Re-complex efficiently reduced CAIXmediated acidification of extracellular medium in vitro efficacy. Though tumor to blood activity ratio increased from 0.65 (at $1 \mathrm{~h}$ p.i.) to 1.14 (at 2 h p.i.), biodistribution results showed that ${ }^{99 \mathrm{~m}} \mathrm{Tc}-$ radiolabeled complex had a low uptake in tumor tissue (only $0.13,0.09,0.08$ and $0.05 \% \mathrm{ID} / \mathrm{g}$ at 0.5, 1, 2 and 4 h p.i.). Rami et al. [66] designed and synthesized several CAIX inhibitors, sulfonamide/sulfamide/sulfamate derivatives containing 2- or 5-nitroimidazoles moieties, which target the active site of CAIX. The 5-nitroimidazole series at a concentration of $1 \mathrm{mM}$ could significantly reduce extracellular tumor acidification in HT29 and HeLa cells, which overexpressed CAIX with treatment of hypoxia. Results from in vivo tests showed the tumors of mice bearing HT29 tumor needed longer average time ( 25 days) to reach $4 \times$ starting volume with the treatment of one of CAIX inhibitors, $\mathrm{N}$-[2-(2-methyl-5-nitro-imidazol-1-yl)ethyl] sulfamide, but the tumors needed only 14 days to reach the same volume with the treatment by vehicle alone. These inhibitors could specifically bind to the active site of CAIX and effectively suppress its activity. Radiolabeled inhibitors may be used for diagnostic or therapeutic purpose in CAIX-positive cancer cells.

Bao et al. have recently developed a quantitative in vivo optical imaging method for detection of CAIX as a marker of tumor hypoxia based on a near-infrared fluorescent derivative of the CAIX 


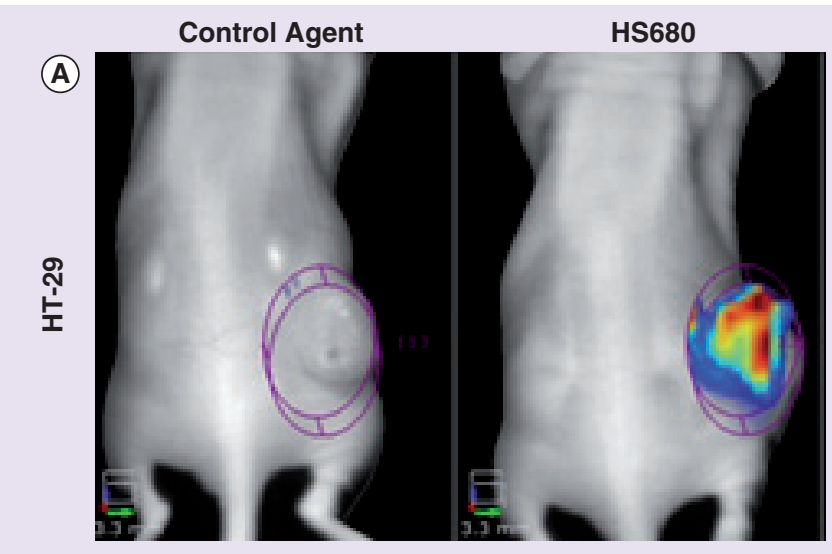

(B)
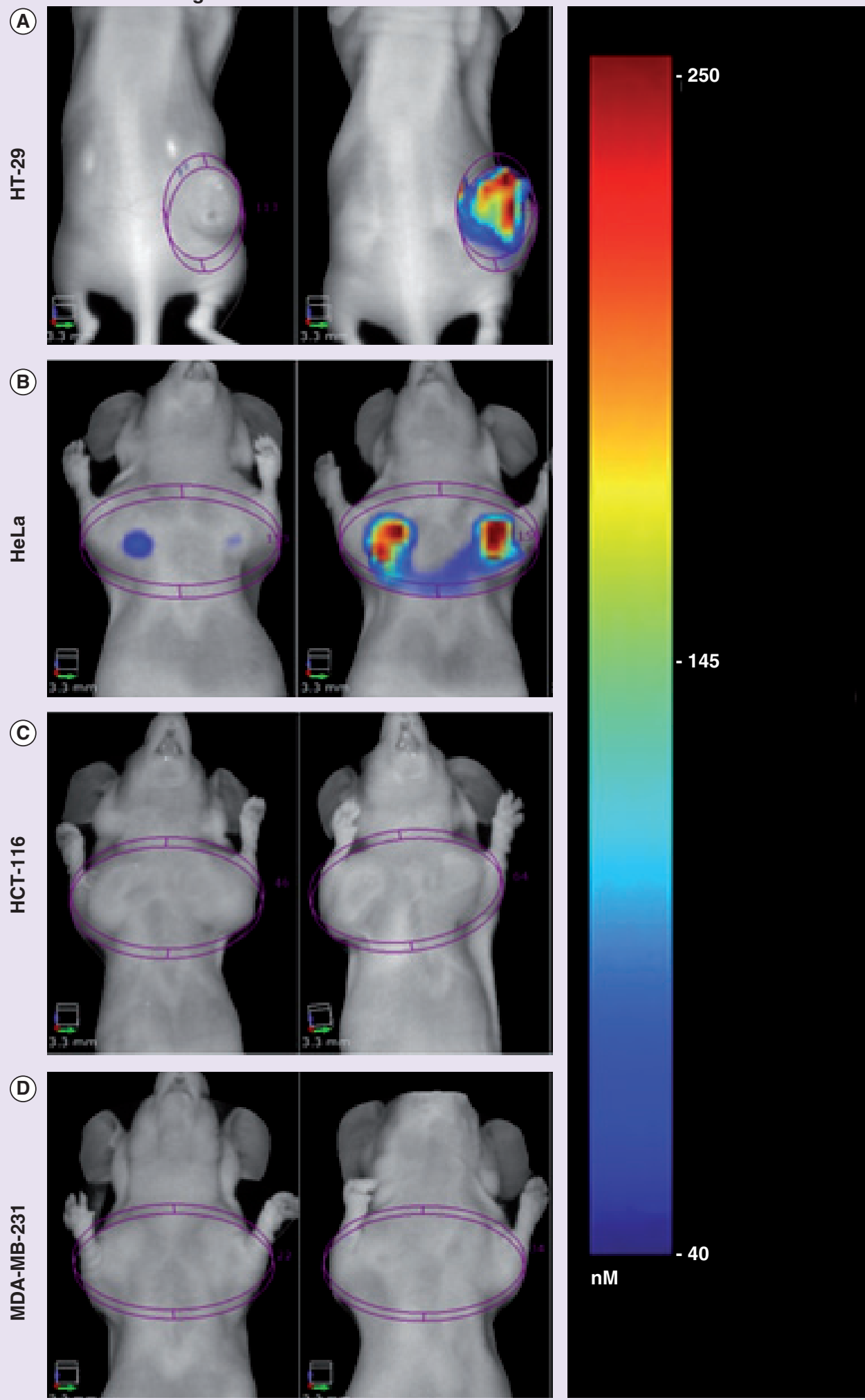

Figure 2. In vivo FMT imaging of HS680 and control agent in mice bearing CAIX-positive (HT-29 and HeLa) and CAIX-negative (HCT-116 and MDA-MB-231) tumors.

For color images please see online at www.futuremedicine.com/doi/full/10.2217/fon.15.11

Reproduced with permission from [32]. 
inhibitor acetazolamide referred as HS680 [32]. The authors demonstrated that CAIX negative cell lines HCT-116 and MDA-MB-231 xenogrfts showed low-HS680 fluorescent signal, whereas CAIX-positive cell lines such HT29 and HeLa xenografts had significant high fluorescent signal, and the author concluded that the potential of HS680 imaging to noninvasively quantify CAIX expression as a hypoxia biomarker, crucial to the study of the underlying biology of hypoxic tumors and the development and monitoring of novel anticancer therapies (Figure 2). However, in the study, co-localization of HS680, pimonidazole and CAIX has been verified in HT29 tumor sections, but did not investigate in HCT-116 and
MDA-MB-231 xenografts which are assumed to present regions of hypoxia. However, results from this study indicates, to some extent, the cell line dependent feature of CAIX expression in cancer cells, and an example to show the limitation of the use of CAIX inhibitor for tumor hypoxia detection or cancer detection.

\section{CAIX-targeted imaging in clinical applications}

Many sound results have been obtained in preclinical studies, some of which have been translated into clinical applications. cG250, a chimeric variant of G250 greatly diminishing the immunogenicity of the G250 antibody, has been
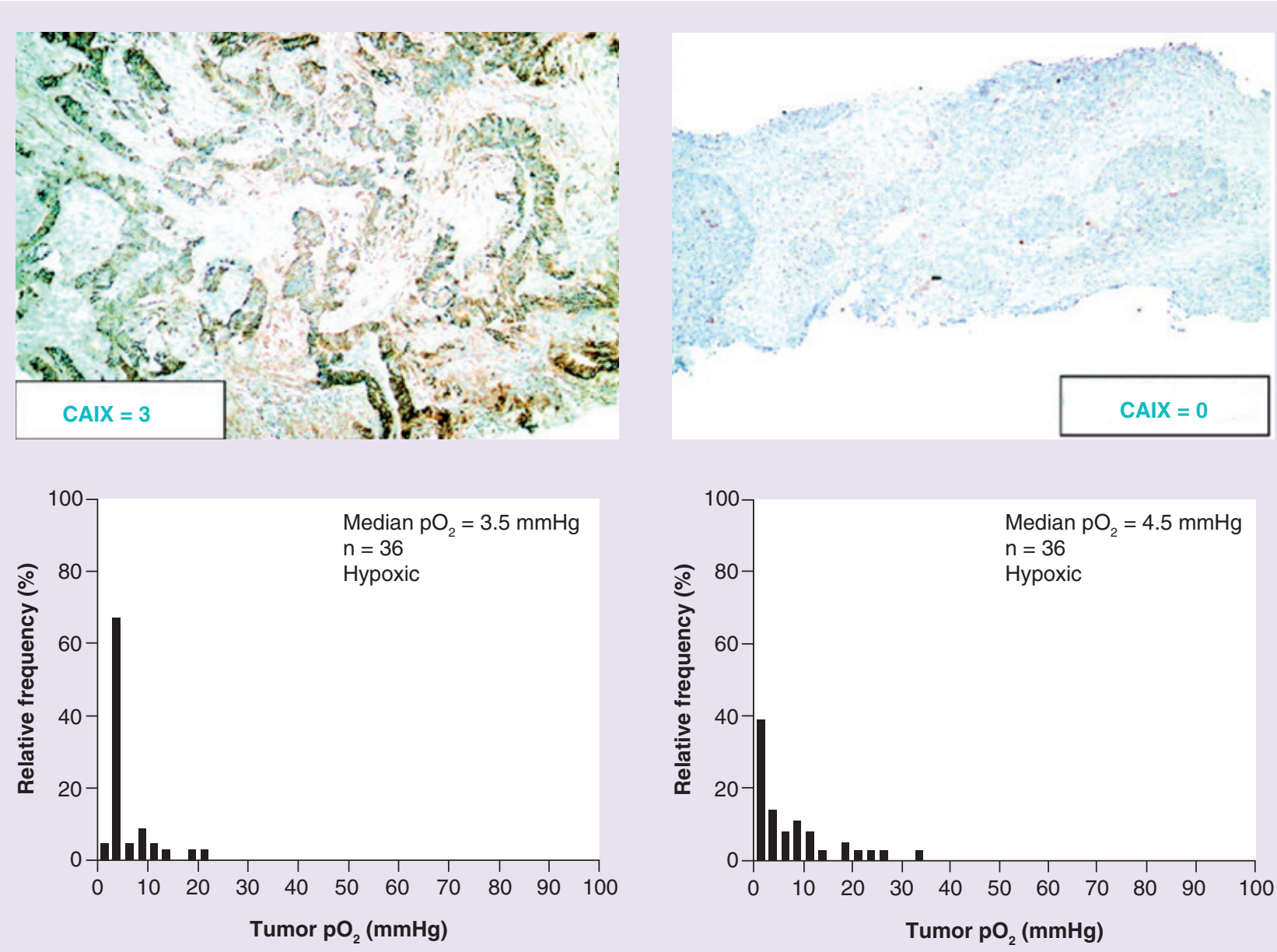

Figure 3. Expression pattern and score of carbonic anhydrase IX with respective oxygen tension $\left(\mathrm{pO}_{2}\right)$. There was strong expression of CAIX in a tumor section on the left upper figure, but there was almost no expression of CAIX in a tumor section shown on the right upper figure; both tumors had median $\mathrm{pO}_{2}$ less than $10 \mathrm{mmHg}$ (hypoxia). This figure was reproduced with permission from Mayer $\mathrm{A}$, Höckel M, Vaupel P: Carbonic anhydrase IX expression and tumor oxygenation status do not correlate at the microregional level in locally advanced cancers of the uterine cervix.

CAIX: Carbonic anhydrase IX.

Reproduced with permission from [35] ๑ by American Association for Cancer Research (2005). 
used in clinical studies [42,67-68]. Pryma et al. conducted PET-CT scans in 26 patients with radiolabeled chimeric antibody ${ }^{124}$ I-cG250 [69]. PET-CT imaging results indicated 15 patients were positive for ${ }^{124} \mathrm{I}-\mathrm{cG} 250$ and ten were negative. For all tumors of surgical resection from ${ }^{124}$ I-cG250 positive patients, the results of autoradiography were similar to the distribution of
CAIX expression as showed by immunohistochemistry. ${ }^{124} \mathrm{I}-\mathrm{cG} 250$ could be a good candidate for noninvasively imaging tumors with expression of CAIX. Genega et al. evaluated CAIX expression in 366 primary and metastatic renal neoplasms by immunohistochemical staining, they found that CAIX is more often expressed in clear cell RCC than other subtypes RCC (71

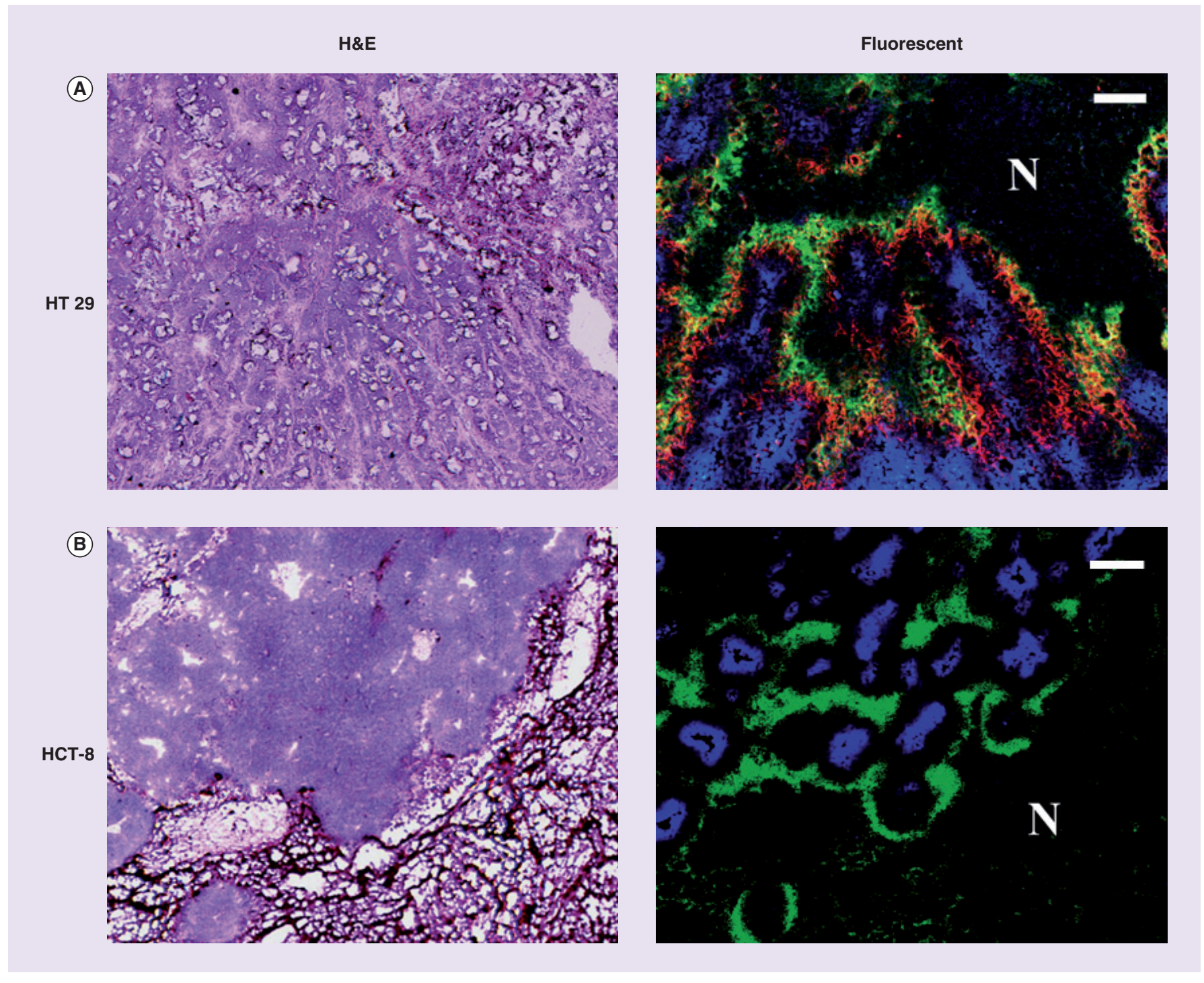

Figure 4. Relationship between carbonic anhydrase IX, pimonidazole and Hoechst 33342 in HT29 and HCT-8 subcutaneous xenografts. (A) H\&E stained section from a macroscopic subcutaneous xenograft derived from the human colorectal carcinoma cell line HT29. Fluorescence overlay image (green = pimonidazole, red = carbonic anhydrase IX [CAIX], blue = Hoechst 33342) shows that there is broad concordance between pimonidazole and CAIX distributions. In particular all identifiable regions of enhanced pimonidazole binding have corresponding regions of enhanced CAIX expression. However, there are regions of CAIX positivity where pimonidazole binding is not seen. Scale bar, $200 \mu \mathrm{m}$. (B) H\&E stained section from a macroscopic subcutaneous xenograft derived from the human colorectal carcinoma cell line HCT-8. Fluorescence overlay image (green = pimonidazole, blue $=$ Hoechst 33342) of the identical section shown on the left. Similarly to the HT29 model, pimonidazole and Hoechst 33342 positivity appear mutually exclusive. However, CAIX is undetectable in HCT-8 tumor section where pimonidazole is stained positively. Scale bar: $200 \mu \mathrm{m}$.

Reproduced with permission from [36]. 


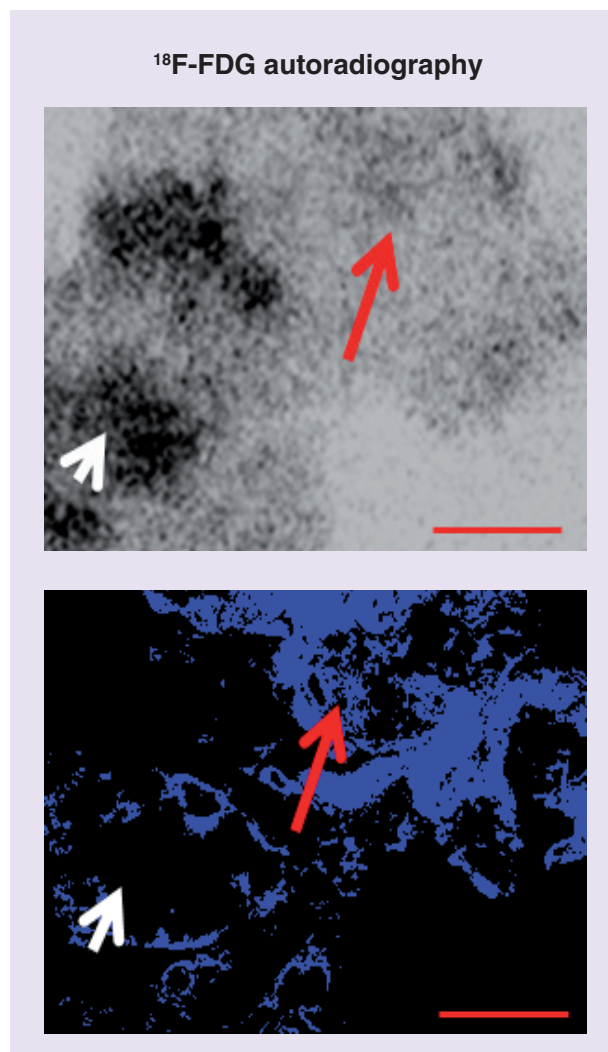

Hoechst 33342
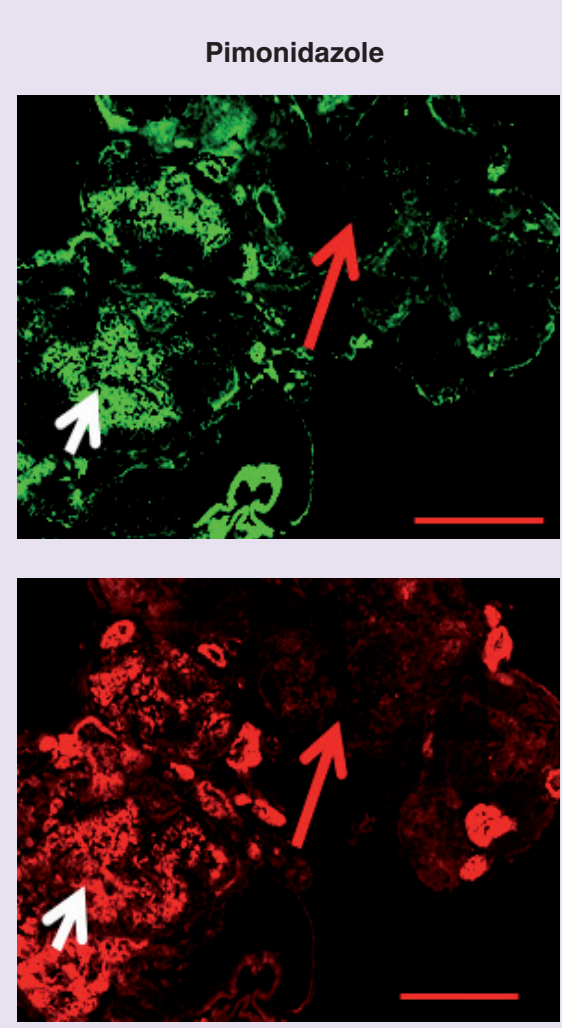

Carbonic anhydrase IX

\section{Bromodeoxyuridine}
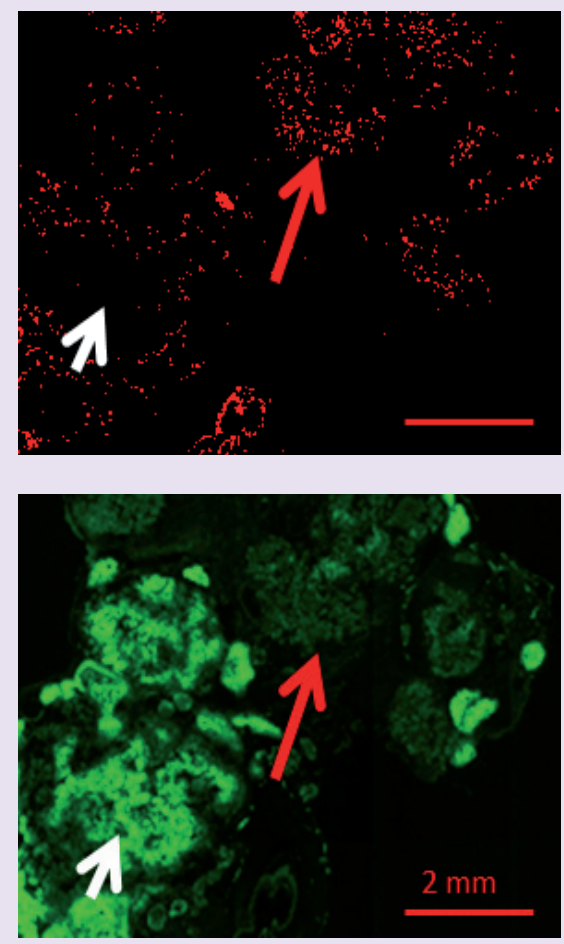

GLUT-1

Figure 5. Relationship between ${ }^{18} \mathrm{~F}$-FDG uptake and hypoxia, proliferation and perfusion in HT29 serosal carcionomatosis hypoxic regions, where pimonidazole, GLUT-1 and carbonic anhydrase IX are stained positively, have high ${ }^{18} \mathrm{~F}-\mathrm{FDG}$ uptake (white arrow). Well perfused regions where Hoechst 33342 is positive and proliferating cancer cells are stained positively for bromodeoxyuridine have low ${ }^{18}$ F-FDG uptake (red arrow). All scale bars: $2 \mathrm{~mm}$.

Reproduced with permission from [74].

vs 3\%), accordingly, targeting CAIX imaging seems to be useful for distinguishing clear cell RCC from other RCC subtypes, but, apparently, it is unlikely to be a gold standard for RCC diagnosis [70].

\section{Limitation of CAIX-targeted imaging}

In selected cancer cell lines, such as HT29 and HeLa cells, preclinical studies have documented that CAIX is a great target for cancer imaging and imaging tumor hypoxia. However, clinical studies indicate that CAIX is overexpressed in some cancer cells but not under conditions of hypoxia. CAIX is unlikely a good target for tumor hypoxia imaging and cancer detection.

Adams et al. analyzed 30,216 immunohistochemistry results from 117 articles and found there were only $35 \%$ expression rates of CAIX in human invasive breast cancer [71]. Mayer et al. found the presence of high CAIX expression in hypoxic regions of some cancers but absence in the others (Figure 3) [35]. And we also documented that CAIX overexpression was similar to pimonidazole binding (exogenous hypoxic marker, specific when $\mathrm{pO}_{2}<10 \mathrm{mmHg}$ ) in colon cancer HT29 xenografts growing in nude mice, while there was no immunohistochemical-stainingdetectable CAIX expression in regions where pimonidazole was stained positively in the rectal cancer HCT-8 xenografts (Figure 4).

Hendrickx et al. [72] conducted a therapeutic clinical trial on targeting CAIX of biliary cancer in three patients using ${ }^{111} \mathrm{In}-\mathrm{cG} 250$ and ${ }^{131} \mathrm{I}$ cG250, respectively. Results from scintigrams of the abdomen of the patient either 5 days after injection of ${ }^{111}$ In-cG250 or 5 days after injection of ${ }^{131} \mathrm{I}-\mathrm{cG} 250$, showed no apparent accumulation of radiolabeled cG250 in tumors. Immunohistochemical analyses revealed the expression of CAIX in tumor tissues of all three 
patients (strong expression for two, moderate for one). So it is inappropriate that cG250, whether labeled with ${ }^{131} \mathrm{I}$ or ${ }^{111} \mathrm{In}$, is used to target tumor tissues in patients bearing biliary cancer.

PET tracer 2-deoxy-2- $\left[{ }^{18} \mathrm{~F}\right]$-fluoro-D-glucose $\left({ }^{18} \mathrm{~F}-\mathrm{FDG}\right)$ has better performance for imaging cancers, we and others had demonstrated that ${ }^{18}$ F-FDG uptake was hypoxia-dependent (Figure 5) [21,73-76]. In addition, hypoxic portion of colorectal cancer HCT-8 xenografts had increased ${ }^{18} \mathrm{~F}$-FDG accumulation [21], but CAIX expression in hypoxic zones was undetectable by immunohistochemistry [36]. ${ }^{18} \mathrm{~F}-\mathrm{FDG}$ is much better PET tracer for cancer detection than targeting CAIX imaging strategies; approximately $95 \%$ of solid malignancies contain hypoxic cancer cells. The advantage of CAIX imaging strategies is that targeting CAIX imaging can be useful for screening purpose to provide information which patients are eligible for targeting CAIX therapy.

With respect to hypoxia imaging, there are few studies to compare CAIX targeted imaging strategies with ${ }^{18} \mathrm{~F}$-fluromisonidazole $\left({ }^{18} \mathrm{~F}-\mathrm{FMISO}\right)$ PET, which may be an interesting project to further explore the limitation of CAIX targeted imaging. Although targeted
CAIX nuclear imaging has be documented, radiolabeled anti-CAIX antibodies autoradiography has compared with immunohistochemical visualization of CAIX expression, whereas a comparison with hypoxia marker pimonidazole (detecting cancer cells when pO2 $<10 \mathrm{mmHg}$ ) immunohistochemical staining has been rarely performed.

\section{Conclusion}

Although succeed in several types of cancer, such as in colorectal cancer HT29 xenografts growing in rodents, CAIX seems unlikely to serve as a universal target for cancer detection or tumor hypoxia visualization. The clinical use of antibodies, probes or inhibitors which targeted CAIX may be limited and unreliable for cancer and hypoxia visualization. However, imaging CAIX may be used to select candidates for anti-CAIX therapies.

\section{Future perspective}

CAIX expression under hypoxic conditions is cell line dependent, targeted CAIX imaging may not be universal hypoxia imaging strategies, and, therefore, would be limited for clinical practice. However, CAIX overexpression may

\section{EXECUTIVE SUMMARY}

\section{Background}

- The presence of hypoxia is a general feature of most solid malignancies and hypoxia is considered as one of major factors for anticancer therapy failure.

- Carbonic anhydrase IX (CAIX) has been reported to be an endogenous hypoxia marker, CAIX monoclonal antibodies, their segments and inhibitors are developed for CAIX imaging.

\section{CAIX}

- CAIX is a membrane-associated glycoprotein, consisting of an extracellular catalytic domain, a proteoglycan-like region (PG domain), a transmembrane anchor and a short C-terminal cytoplasmic tail.

- The overexpression of CAIX in normal human tissues is rare.

- CAIX regulates intracellular $\mathrm{pH}$.

\section{CAIX \& tumor hypoxia}

- CAIX has been considered as an endogenous hypoxic marker.

- CAIX expression under hypoxic conditions may be dependent on cancer cell line or cancer type.

- CAIX may be unreliable as a universal target for cancer and tumor hypoxia visualization; CAIX imaging may identify patients that are likely to benefit from targeting CAIX therapy.

\section{Future perspective}

- CAIX overexpression may play critical role on prediction anticancer therapeutic effect.

- The role of CAIX in addition to hypoxia in oncology should be addressed. 
play a critical role in the prediction of anticancer therapeutic effect. CAIX overexpression is not universal in hypoxic cancer cells. Investigations on the outcome and progression CAIX-positive hypoxic cancer versus CAIX-negative hypoxic cancer should be conducted.

\section{Financial \& competing interests disclosure}

The authors' work is supported in part by the Kentucky Lung Cancer Research program award (cycle9), Inner Mongolia major basic science research program award (to hypoxic microenviroment of lung cancer metastases and hypoxia targeted therapy), National Natural Science Foundation of China award (81360227), Inner Mongolia Natural Science Foundation awards (2013MS1188), and NIH grants R01 CA84596. The authors have no other relevant affiliations or financial involvement with any organization or entity with a financial interest in or financial conflict with the subject matter or materials discussed in the manuscript apart from those disclosed.

No writing assistance was utilized in the production of this manuscript.

\section{References}

Papers of special note have been highlighted as: - of interest; $\bullet \bullet$ of considerable interest

1 Siegel R, Naishadham D, Jemal A. Cancer statistics, 2013. CA Cancer J. Clin. 63(1), 11-30 (2013).

2 Thomlinson RH, Gray LH. The histological structure of some human lung cancers and the possible implications for radiotherapy. $\mathrm{Br}$. J. Cancer 9(4), 539-549 (1955).

3 Höckel M, Schlenger K, Knoop C, Vaupel P. Oxygenation of carcinomas of the uterine cervix: evaluation by computerized $\mathrm{O}_{2}$ tension measurements. Cancer Res. 51(22), 6098-6102 (1991).

4 Höckel M, Knoop C, Schlenger K, Vorndran B, Knapstein PG, Vaupel P. Intratumoral pO2 histography as predictive assay in advanced cancer of the uterine cervix. $A d v$. Exp. Med. Biol. 345, 445-450 (1994).

5 Brizel DM, Lin S, Johnson JL, Brooks J, Dewhirst MW, Piantadosi CA. The mechanisms by which hyperbaric oxygen and carbogen improve tumour oxygenation. Br. J. Cancer 72(5), 1120-1124 (1995).

6 Chapman JD, Engelhardt EL, Stobbe CC, Schneider RF, Hanks GE. Measuring hypoxia and predicting tumor radioresistance with nuclear medicine assays. Radiother. Oncol. 46(3), 229-237 (1998).

7 Wouters BG, Weppler SA, Koritzinsky M et al. Hypoxia as a target for combined modality treatments. Eur. J. Cancer 38(2), 240-257 (2002).

8 Koch CJ, Evans SM. Non-invasive PET and SPECT imaging of tissue hypoxia using isotopically labeled 2-nitroimidazoles. $A d v$. Exp. Med. Biol. 510, 285-292 (2003).

9 Goethals L, Debucquoy A, Perneel C et al. Hypoxia in human colorectal adenocarcinoma: comparison between extrinsic and potential intrinsic hypoxia markers. Int. J. Radiat. Oncol. Biol. Phys. 65(1), 246-254 (2006).
10 Brizel DM, Scully SP, Harrelson JM et al. Tumor oxygenation predicts for the likelihood of distant metastases in human soft tissue sarcoma. Cancer Res. 56(5), 941-943 (1996).

11 Brizel DM, Sibley GS, Prosnitz LR, Scher RL, Dewhirst MW. Tumor hypoxia adversely affects the prognosis of carcinoma of the head and neck. Int. J. Radiat. Oncol. Biol. Phys. 38(2), 285-289 (1997).

12 Graeber TG, Osmanian C, Jacks T et al. Hypoxia-mediated selection of cells with diminished apoptotic potential in solid tumours. Nature 379 (6560), 88-91 (1996).

13 Rofstad EK. Microenvironment-induced cancer metastasis. Int. J. Radiat. Biol. 76(5), 589-605 (2000).

- The hypoxic environment is selective for genetically unstable tumor cells, and hypoxia is associated with tumors that are more likely to metastasize.

14 Semenza GL. Regulation of mammalian O2 homeostasis by hypoxia-inducible factor 1 . Annu. Rev. Cell Dev. Biol. 15, 551-578 (1999).

15 Tang CM, Yu J. Hypoxia-inducible factor-1 as a therapeutic target in cancer. J. Gastroenterol. Hepatol. 28(3), 401-405 (2013).

16 Gilkes DM, Semenza GL. Role of hypoxiainducible factors in breast cancer metastasis. Future Oncol. 9(11), 1623-1636 (2013).

17 Ghattass K, Assah R, El-Sabban M, Gali-Muhtasib H. Targeting hypoxia for sensitization of tumors to radio- and chemotherapy. Curr. Cancer Drug Targets 13(6), 670-685 (2013).

18 Ellis LM, Hicklin DJ. VEGF-targeted therapy: mechanisms of anti-tumour activity. Nat. Rev. Cancer 8(8), 579-591 (2008).

19 Conti A, Santoni M, Amantini C et al. Progress of molecular targeted therapies for advanced renal cell carcinoma. Biomed. Res. Int. 2013, 9 (2013).
20 Fakhrejahani E, Toi M. Antiangiogenesis therapy for breast cancer: an update and perspectives from Clinical trials. Jpn. J. Clin. Oncol. 44(3), 197-207 (2014).

21 Li XF, Ma Y, Sun X, Humm JL, Ling CC, O'Donoghue JA. High 18F-FDG uptake in microscopic peritoneal tumors requires physiologic hypoxia. J. Nucl. Med. 51(4), 632-638 (2010).

-• It demonstrated that 18F-FDG uptake was hypoxia-dependent, and 18F-FDG has better performance for imaging cancers.

22 Szablewski L. Expression of glucose transporters in cancers. Biochim. Biophys. Acta 1835(2), 164-169 (2012).

23 Huang T, Civelek AC, Zheng $\mathrm{H}$ et al. $18 \mathrm{~F}$-misonidazole PET imaging of hypoxia in micrometastases and macroscopic xenografts of human non-small cell lung cancer: a correlation with autoradiography and histological findings. Am. J. Nucl. Med. Mol. Imaging 3(2), 142-153 (2013).

$24 \mathrm{Li}$ J, Shi L, Wang C et al. Preliminary biological evaluation of 125I-labeled anti-carbonic anhydrase IX monoclonal antibody in the mice bearing HT-29 tumors. Nucl. Med. Commun. 32(12), 1190-1193 (2011).

25 Mcdonald PC, Winum JY, Supuran CT, Dedhar S. Recent developments in targeting carbonic anhydrase IX for cancer therapeutics. Oncotarget 3(1), 84-97 (2012).

26 Proescholdt MA, Merrill MJ, Stoerr EM, Lohmeier A, Pohl F, Brawanski A. Function of carbonic anhydrase IX in glioblastoma multiforme. Neuro Oncol. 14(11), 1357-1366 (2012).

27 Gieling RG, Williams KJ. Carbonic anhydrase IX as a target for metastatic disease. Bioorg. Med. Chem. 21(6), 1470-1476 (2013).

28 Tafreshi NK, Lloyd MC, Bui MM, Gillies RJ, Morse DL. Carbonic anhydrase IX as an imaging and therapeutic target for tumors 
and metastases. Subcell Biochem. 75, 221-254 (2014).

29 Benej M, Pastorekova S, Pastorek J. Carbonic anhydrase IX: regulation and role in cancer. Subcell Biochem. 75, 199-219 (2014).

30 Ivanov S, Liao SY, Ivanova A et al. Expression of hypoxia-inducible cell-surface transmembrane carbonic anhydrases in human cancer. Am. J. Pathol. 158(3), 905-919 (2001).

31 Rademakers SE, Span PN, Kaanders JH, Sweep FC, Van Der Kogel AJ, Bussink J. Molecular aspects of tumour hypoxia. Mol. Oncol. 2(1), 41-53 (2008).

32 Bao B, Groves K, Zhang J et al. In vivo imaging and quantification of carbonic anhydrase IX expression as an endogenous biomarker of tumor hypoxia. PLoS ONE 7(11), e50860 (2012).

33 Carlin S, Khan N, Ku T, Longo VA, Larson SM, Smith-Jones PM. Molecular targeting of carbonic anhydrase IX in mice with hypoxic HT29 colorectal tumor xenografts. PLoS ONE 5(5), e10857 (2010).

34 Mcdonald PC, Dedhar S. Carbonic anhydrase IX (CAIX) as a mediator of hypoxia-induced stress response in cancer cells. Subcell Biochem. 75, 255-269 (2014).

35 Mayer A, Höckel M, Vaupel P. Carbonic anhydrase IX expression and tumor oxygenation status do not correlate at the microregional level in locally advanced cancers of the uterine cervix. Clin. Cancer Res. 11(20), 7220-7225 (2005).

-• It shows some cancer cell lines have undetectable CAIX expression under hypoxia condition. They raised doubts concerning whether it was suitable for CAIX as a substitute for oxygen microelectrode measurements and as an endogenous hypoxia marker. Because the expression of CAIX in vivo was not only associated with the state of tumor hypoxia, but also with a number of other factors, it was not applicable for CAIX to image tumor hypoxia in clinical trials

36 Li XF, Carlin S, Urano M, Russell J, Ling CC, O'Donoghue JA. Visualization of hypoxia in microscopic tumors by immunofluorescent microscopy. Cancer Res. 67(16), 7646-7653 (2007).

37 Imtaiyaz Hassan M, Shajee B, Waheed A, Ahmad F, Sly WS. Structure, function and applications of carbonic anhydrase isozymes. Bioorg. Med. Chem. 21(6), 1570-1582 (2013).

38 Pastoreková S, Závadová Z, Koš ál M, Babušíková O, Závada J. A novel quasi-viral agent, $\mathrm{MaTu}$, is a two-component system. Virology 187(2), 620-626 (1992).
39 Pastorek J, Pastorekova S, Callebaut I et al. Cloning and characterization of $\mathrm{MN}$, a human tumor-associated protein with a domain homologous to carbonic anhydrase and a putative helix-loop-helix DNA binding segment. Oncogene 9(10), 2877-2888 (1994).

40 Thiry A, Dogne JM, Masereel B, Supuran CT. Targeting tumor-associated carbonic anhydrase IX in cancer therapy. Trends Pharmacol. Sci. 27(11), 566-573 (2006).

- CAIX has limited expression and distribution in a few normal tissues (mainly the GI tract), however, it is ectopically expressed in numerous tumors which are mostly derived from tissues that do not normally express CAIX.

41 Chen J, Röcken C, Hoffmann J et al. Expression of carbonic anhydrase 9 at the invasion front of gastric cancers. Gut 54(7), 920-927 (2005).

42 Stillebroer AB, Mulders PF, Boerman OC, Oyen WJ, Oosterwijk E. Carbonic anhydrase IX in renal cell carcinoma: implications for prognosis, diagnosis, and therapy. Eur. Urol. 58(1), 75-83 (2010).

43 De Simone G, Supuran CT. Carbonic anhydrase IX: biochemical and crystallographic characterization of a novel antitumor target. Biochim. Biophys. Acta 1804(2), 404-409 (2010).

44 Parks SK, Chiche J, Pouyssegur J. pH control mechanisms of tumor survival and growth. J. Cell. Physiol. 226(2), 299-308 (2011).

45 Švastová E, Žilka N, Zat'ovi ová M et al. Carbonic anhydrase IX reduces E-cadherinmediated adhesion of MDCK cells via interaction with $\beta$-catenin. Exp. Cell Res. 290(2), 332-345 (2003).

46 Glazer PM, Hegan DC, Lu Y, Czochor J, Scanlon SE. Hypoxia and DNA Repair. Yale J. Biol. Med. 86(4), 443-451 (2013).

47 Taiakina D, Dal Pra A, Bristow RG. Intratumoral hypoxia as the genesis of genetic instability and clinical prognosis in prostate cancer. Adv. Exp. Med. Biol. 772, 189-204 (2014).

48 Bittner MI, Grosu AL. Hypoxia in head and neck tumors: characteristics and development during therapy. Front. Oncol. 3, 223 (2013).

49 Ilie M, Mazure NM, Hofman V et al. High levels of carbonic anhydrase IX in tumour tissue and plasma are biomarkers of poor prognostic in patients with non-small cell lung cancer. $B r$. J. Cancer 102(11), 1627-1635 (2010).

50 Said HM, Supuran CT, Hageman C et al. Modulation of carbonic anhydrase 9 (CA9) in human brain cancer. Curr. Pharm. Des. 16(29), 3288-3299 (2010).
51 Rundqvist H, Johnson RS. Tumour oxygenation: implications for breast cancer prognosis. J. Intern. Med. 274(2), 105-112 (2013).

52 Brahimi-Horn MC, Pouysségur J. Oxygen, a source of life and stress. FEBS Lett. 581(19), 3582-3591 (2007).

53 Huang LE, Bindra RS, Glazer PM, Harris AL. Hypoxia-induced genetic instability-a calculated mechanism underlying tumor progression. J. Mol. Med. (Berlin). 85(2), 139-148 (2007).

54 Ruan K, Song G, Ouyang G. Role of hypoxia in the hallmarks of human cancer. J. Cell. Biochem. 107(6), 1053-1062 (2009).

55 Semenza GL. HIF-1 mediates metabolic responses to intratumoral hypoxia and oncogenic mutations. J. Clin. Invest. 123(9), 3664-3671 (2013).

56 Yang Y, Sun M, Wang L, Jiao B. HIFs, angiogenesis, and cancer. J. Cell. Biochem. 114(5), 967-974 (2013).

57 Kaluz S, Kaluzová M, Stanbridge EJ. Regulation of gene expression by hypoxia: integration of the HIF-transduced hypoxic signal at the hypoxia-responsive element. Clin. Chim. Acta 395(1-2), 6-13 (2008).

58 Kizaka-Kondoh S, Tanaka S, Harada H, Hiraoka M. The HIF-1-active microenvironment: an environmental target for cancer therapy. Adv. Drug Deliv. Rev. 61(7-8), 623-632 (2009).

59 Monti SM, Supuran CT, De Simone G. Carbonic anhydrase IX as a target for designing novel anticancer drugs. Curr. Med. Chem. 19(6), 821-830 (2012).

60 Ljungkvist AS, Bussink J, Kaanders JH et al. Hypoxic cell turnover in different solid tumor lines. Int. J. Radiat. Oncol. Biol. Phys. 62(4), 1157-1168 (2005).

61 Gifford GE. Some effects of anaerobiosis on the growth and metabolism of HeLa cells. Exp. Cell Res. 31, 113-118 (1963).

62 Littbrand B, Révész L. Survival of cells in anoxia. Br. J. Radiol. 41(486), 479-480 (1968).

63 Koch CJ, Kruuv J, Frey HE. The effect of hypoxia on the generation time of mammalian cells. Radiat. Res. 53(1), 43-48 (1973).

64 Koch CJ, Kruuv J, Frey HE, Snyder RA. Plateau phase in growth induced by hypoxia. Int. J. Radiat. Biol. Relat. Stud. Phys. Chem. Med. 23(1), 67-74 (1973).

65 Akurathi V, Dubois L, Lieuwes NG et al. Synthesis and biological evaluation of a 99mTc-labelled sulfonamide conjugate for 
in vivo visualization of carbonic anhydrase IX expression in tumor hypoxia. Nucl. $\mathrm{Med}$. Biol. 37(5), 557-564 (2010). Rami M, Dubois L, Parvathaneni NK et al. Hypoxia-targeting carbonic anhydrase IX inhibitors by a new series of nitroimidazolesulfonamides/sulfamides/sulfamates. J. Med. Chem. 56(21), 8512-8520 (2013).

Davis ID, Wiseman GA, Lee FT et al. A phase I multiple dose, dose escalation study of cG250 monoclonal antibody in patients with advanced renal cell carcinoma. Cancer Immun. 7, 13 (2007).

68 Muselaers S, Mulders P, Oosterwijk E, Oyen W, Boerman O. Molecular imaging and carbonic anhydrase IX-targeted radioimmunotherapy in clear cell renal cell carcinoma. Immunotherapy 5(5), 489-495 (2013).

69 Pryma DA, 'donoghue JA, Humm JL et al. Correlation of in vivo and in vitro measures of carbonic anhydrase IX antigen expression in renal masses using antibody ${ }^{124} \mathrm{I}-\mathrm{cG} 250$. J. Nucl. Med. 52(4), 535-540 (2011).

Genega EM, Ghebremichael M, Najarian R et al. Carbonic anhydrase IX expression in renal neoplasms correlation with tumor type and grade. Am. J. Clin. Pathol. 134(6), 873-879 (2010).

71 Adams A, Van Brussel AS, Vermeulen JF et al. The potential of hypoxia markers as target for breast molecular imaging - a systematic review and meta-analysis of human marker expression. BMC Cancer 13, 538 (2013).

72 Hendrickx BW, Punt CJ, Boerman OC et al. Targeting of biliary cancer with radiolabeled chimeric monoclonal antibody CG250. Cancer Biother. Radiopharm. 21(3), 263-268 (2006).

73 Huang T, Civelek AC, Li J et al. Tumor microenvironment-dependent ${ }^{18} \mathrm{~F}-\mathrm{FDG}$, ${ }^{18} \mathrm{~F}$-fluorothymidine, and $18 \mathrm{~F}$-misonidazole uptake: a pilot study in mouse models of human non-small cell lung cancer. J. Nucl. Med. 53(8), 1262-1268 (2012).

74 Li XF, Du Y, Ma Y, Postel GC, Civelek AC. ${ }^{18}$ F-fluorodeoxyglucose uptake and tumor hypoxia: revisit ${ }^{18} \mathrm{~F}$-fluorodeoxyglucose in oncology application. Transl. Oncol. 7(2), 240-247 (2014).

- It shows relationship between ${ }^{18}$ F-FDG uptake and hypoxia, proliferation and perfusion in HT29 serosal carcionomatosis.

75 Pugachev A, Ruan S, Carlin S et al. Dependence of FDG uptake on tumor microenvironment. Int. J. Radiat. Oncol. Biol. Phys. 62(2), 545-553 (2005).

-. It demonstrated that ${ }^{18} \mathrm{~F}-\mathrm{FDG}$ uptake was hypoxia-dependent.

76 Zhang G, Li J, WangX et al. The reverseWarburg effect and 18 F-FDG uptake in non-small cell lung cancer A549 in mice: apilot study. J. Nucl. Med. 56(4), 607-612 (2015). 